\title{
Validation of Fanconi anemia complementation Group A assignment using molecular analysis
}

\author{
Nabil N. Moghrabi, PhD $D^{1}$, Monique A. Johnson, PhD $D^{I}$, Marvin J. Yoshitomi, BS $S^{l}$ Xiaoman Zhu, MD', \\ Muhsen J. Al-Dhalimy, MSc ${ }^{1}$, Susan B. Olson, PhD ${ }^{1}$, Markus Grompe, $M D^{2,3}$, \\ and C. Sue Richards, $P h D^{I}$
}

\begin{abstract}
Purpose: Fanconi anemia is a genetically heterogeneous chromosomal breakage disorder exhibiting a high degree of clinical variability. Clinical diagnoses are confirmed by testing patient cells for increased sensitivity to crosslinking agents. Fanconi anemia complementation group assignment, essential for efficient molecular diagnosis of the disease, had not been validated for clinical application before this study. The purpose of this study was (1) confirmation of the accuracy of Fanconi anemia complementation group assignment to Group A (FANCA) and (2) development of a rapid mutation detection strategy that ensures the efficient capture of all FANCA mutations. Methods: Using fibroblasts from 29 patients, diagnosis of Fanconi anemia and assignment to complementation Group A was made through breakage analysis studies. FANCA coding and flanking sequences were analyzed using denaturing high pressure liquid chromatography, sequencing, and multiplex ligation-dependent probe amplification. Patients in which two mutations were not identified were analyzed by cDNA sequencing. Patients with no mutations were sequenced for mutations in FANCC, G, $E$, and $F$. Results: Of the 56 putative mutant alleles studied, $89 \%$ had an identifiable $F A N C A$ pathogenic mutation. Eight unique novel mutations were identified. Conclusion: Complementation assignment to Group A was validated in a clinical laboratory setting using our FANCA rapid molecular testing strategy. Genet Med 2009:11(3):183-192.
\end{abstract}

Key Words: Fanconi anemia, molecular FANCA testing, FANCA, complementation group assignment

Eanconi anemia (FA; MIM no. 227650), the most common Finherited bone marrow disorder, has an overall prevalence of 1-5 per million and an estimated carrier frequency of 1 in 200 to 1 in 300 in most populations. ${ }^{1,2}$ Demonstrating either an autosomal or X-linked recessive mode of inheritance, FA is characterized by childhood progressive bone marrow failure and predisposition to acute myelogenous leukemia; older patients are at increased risk for squamous cell carcinomas of the head, neck, and genitourinary tract. ${ }^{3-7}$ Congenital abnormalities are present in approximately $70 \%$ of FA patients and may include radial ray defects; café au lait spots or hypopigmentation; short stature; microphthalmia; malformations of kidneys,

From the ${ }^{1}$ Department of Molecular and Medical Genetics, Clinical Genetics Laboratories, ${ }^{2}$ Oregon Stem Cell Center, and ${ }^{3}$ Department of Pediatrics, Oregon Health \& Science University, Portland, Oregon.

C. Sue Richards, PhD, Department of Molecular and Medical Genetics, Clinical Genetics Laboratories, Mail Code: MP350, 3181 S.W. Sam Jackson Park Road, Portland, OR 97239. E-mail: richarsu@ohsu.edu.

Supplemental digital content is available for this article. Direct URL citations appear in the printed text and are provided in the HTML and PDF versions of this article on the journal's Web site (www.geneticsinmedicine.org).

Disclosure: The authors declare no conflict of interest.

Submitted for publication June 5, 2008.

Accepted for publication October 30, 2008.

DOI: $10.1097 /$ GIM.0b013e318193ba67 gastrointestinal tract, and heart; mental retardation; and hearing defects. ${ }^{8}$

Because of the high degree of phenotypic variability exhibited by FA patients, diagnosis may be difficult on the basis of clinical manifestations alone. Because FA patient-derived lymphocytes and fibroblasts exhibit hypersensitivity to DNA crosslinking agents such as diepoxybutane (DEB) ${ }^{9}$ and mitomycin C (MMC), ${ }^{10}$ resulting in a high rate of chromosomal breakage and radial formation, analysis on the basis of this hypersensitivity has been routinely used to confirm clinical diagnosis.

Molecular diagnosis of FA has been challenging because of the genetic heterogeneity associated with the disease; FA is multigenic, with 13 complementation groups and associated genes having been characterized (A, B, C, D1, D2, E, F, G, I, J, $\mathrm{L}, \mathrm{M}, \mathrm{N}) .{ }^{11-27}$ Cloning of the $F A N C$ genes has provided a means, via complementation group analysis, to routinely pinpoint the patient's specific FA gene harboring the mutations, thus greatly simplifying subsequent molecular analysis. ${ }^{28,29}$

Although retroviral-mediated complementation analysis of FA patient-derived cells is commonly used to identify the patient's genetic subtype as a prerequisite for mutation screening, such analysis has not been formally validated for clinical use. Previous studies have considered Fanconi complementation Group A (FA-A) assignments, but none have performed comprehensive sequencing to verify complementation assignment and/or evaluated complementation assignments performed using retroviral-mediated rescue as evidenced by correction of MMC and DEB-induced chromosome breakage and radial formation. ${ }^{29-32}$

Mutations in the gene for complementation group FA-A (FANCA; MIM no. 607139) confer autosomal recessive inheritance and account for approximately $66 \%$ of all FA cases. ${ }^{33}$ The FANCA gene is mapped to chromosome $16 \mathrm{q} 24.3,{ }^{11}$ spans approximately $80 \mathrm{~kb}$ of genomic DNA (gDNA), and consists of 43 exons. ${ }^{34}$ With more than 200 different mutations described thus far, the FANCA mutation spectrum is very

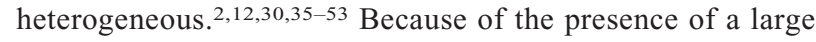
number of $A l u$ repeat sequences within the FANCA gene, large intragenic deletions involving multiple exons have been reported to account for more than $40 \%$ of all FANCA mutations. ${ }^{54,55}$ Because the majority of FA patients fall into complementation group FA-A, we initiated verification of accuracy of FA complementation assignment in this genetic subtype. As a prerequisite, we first developed a rapid and efficient molecular assay for analysis of FANCA in a diagnostic setting. Because of the high frequency of private mutations, polymorphisms, and gross deletions, as well as the large size of $F A N C A$, our analysis strategy integrates rapid mutation scanning, sequencing, and deletion/duplication testing. Additionally, in this study we report novel mutations identified in our cohort of FA-A patients. 


\section{MATERIALS AND METHODS}

\section{Patients}

A subset of fibroblast samples in the Oregon Health \& Science University (OHSU) Fanconi Anemia Cell Repository represent 29 patients who had previously been diagnosed with FA on the basis of clinical features including progressive pancytopaenia, characteristic congenital abnormalities such as radial defects, skin pigmentation, and slow growth. In all cases, a diagnosis of FA was confirmed cytogenetically and each was assigned to complementation Group A as described below. Informed consent for genetic analysis was obtained from all families, and the study was approved by the Institutional Review Board of OHSU.

\section{DNA, RNA, and cDNA sample preparation}

The fibroblasts were cultured by standard techniques and gDNA was extracted from each of the cell lines using a modified version of an organic extraction protocol. ${ }^{28}$ In short, $7 \times$ $10^{5}$ to $2 \times 10^{6}$ fibroblast cells were trypsonized, washed in $1 \times$ phosphate-buffered saline and cell pellets were resuspended in $465 \mu \mathrm{L}$ Nuclei Dropping buffer $(0.075 \mathrm{M} \mathrm{NaCl}, 0.024 \mathrm{M}$ EDTA). Ten-microliter proteinase $\mathrm{K}(10 \mathrm{mg} / \mathrm{mL})$ and $25 \mu \mathrm{L}$ $10 \%$ SDS were added to the suspension mix and was incubated at $37^{\circ} \mathrm{C}$ for 16 hours. This was followed by organic extraction using standard protocol. ${ }^{56}$

Total RNA, enriched for mRNA, was extracted from each of the cell lines (cultured fibroblasts) using the RNeasy Mini Kit (Qiagen, Valencia, CA). A QIAshredder (Qiagen) was used to homogenize the lysate and an RNase-Free DNase Set (Qiagen) was used on-column to digest DNA during the RNA purification.

The resulting RNA was reverse transcribed (RT-PCR [polymerase chain reaction]) to cDNA using a lab developed assay. Briefly, $10 \mu \mathrm{L}$ of RNA was denatured at $65^{\circ} \mathrm{C}$ for 10 minutes and then added to $10 \mu \mathrm{L}$ of the following master mix: 1X RT Buffer (Invitrogen, Carlsbad, CA), $10 \mathrm{mM}$ dithiothreitol, 50 $\mu \mathrm{M}$ Roche $\operatorname{Pd}(\mathrm{N})_{6}$ random primers (Roche, Nutley, NJ), 0.2 $\mathrm{mM}$ each dinucleotide triphosphates (dNTPs), $10 \mathrm{U} / \mu \mathrm{L}$ SuperScript II Polymerase (Invitrogen), and $2 \mathrm{U} / \mu \mathrm{L}$ Roche Protector Rnase Inhibitor (Roche). Amplification was performed in a PTC-200 Peltier Thermal Cycler (MJ Reseach, Waltham, MA) as follows: $25^{\circ} \mathrm{C}$ for 10 minutes, $42^{\circ} \mathrm{C}$ for 50 minutes, $70^{\circ} \mathrm{C}$ for 15 minutes, and $4^{\circ} \mathrm{C}$ hold.

\section{Cytogenetic analysis}

Twenty-nine fibroblasts cell lines, held at the OHSU Fanconi Anemia Cell Repository, were cultured as described previously. ${ }^{28} \mathrm{~A}$ diagnosis of FA was confirmed in the 29 patient-derived fibroblasts cell lines by cytogenetic analysis of sensitivity to MMC (Sigma-Aldrich, St Louis, MO) and DEB (SigmaAldrich) as previously described (Table 1). ${ }^{4,57,58}$ Briefly, approximately 50 Wright-stained metaphase spreads from each of the following cultures were assayed for chromosomal breakage and radial formation: (1) patient-derived DEB (multiple concentrations) and MMC- (multiple concentrations) treated fibroblasts and (2) untreated patient-derived fibroblasts. A diagnosis of FA was confirmed if $\geq 20 \%$ of fibroblasts demonstrated radials. Complementation analysis was performed on the $29 \mathrm{FA}$ patient-derived fibroblasts by retroviral (FANCA encoded) transfection followed by assessment of chromosomal breakage and radial formation. The transfected cell cultures, treated and untreated with MMC and DEB as above, were assayed as previously described. ${ }^{28,29}$ Retroviral mediated correction of hypersensitivity to MMC and DEB was inferred if the percent of chromosome breaks and radials/cell detected was $<10 \%$ using either DEB or MMC. All samples were blinded to us regarding previous genetic results.

\section{Genomic controls}

A total of 19 FANCA gDNA samples harboring known mutations distributed throughout the FANCA gene (see Table, Supplemental Digital Content 1, http://links.lww.com/A777, Positive controls used for $F A N C A$ test development) and four gDNA samples from unaffected individuals were used in this study for test validation of clinical and analytical specificity. $2,12,31,35,36,39,44,47,52,59$

\section{Reference sequences}

The FANCA genomic reference sequence used for genomic primer sequence selection was GenBank accession number NT_010542. The single nucleotide polymorphism (SNP) database used was at NCBI, geneID number $2175 .{ }^{60}$ For numbering of variants and design of cDNA primers, GenBank FANCA cDNA reference sequence was used (accession number NM_000135.2 with +1 corresponding to the A of the ATG translation initiation codon at position $43 \mathrm{bp}$ in the reference sequence). The FANCA gene mutation information sources were published citations and citations at the web-based databases, below. ${ }^{59,61,62}$

\section{Primer and melting curve profile design}

All of the primers used in this study were designed using "OligoAnalyzer 3.0" software (Integrated DNA Technologies, Coralville, IA). This design allowed for the simultaneous and robust amplification of the entire FANCA coding region, using genomic primers, and their flanking sequence as well as for the determination of optimal melting profile of the amplified PCR product for denaturing high pressure liquid chromatography (dHPLC) as determined by "Mutation Discovery" software (Transgenomics, Omaha, NE) (see Table, Supplemental Digital Content 2, http://links.lww.com/A794, FANCA primer sequences; amplicon size; dHPLC parameters (oven temperature, $\%$ B buffer composition)). All primer sequences were tailed with M13 forward and reverse primers to allow for efficient sequencing. In the case of apparent homozygosity in patient samples, alternate primers were designed to rule out allele dropout. PCR for gDNA was performed in $50 \mu \mathrm{L}$ total volume containing $150 \mathrm{ng}$ of gDNA, $15 \mathrm{pmol}$ of each primer, and 10 mM dNTP. Additionally, for FANCA exon 1, the following was added from FastStart PCR kit from Roche (New Jersey): $5 \mu \mathrm{L}$ of $10 \times$ buffer $+\mathrm{MgCl}_{2}, 10 \mu \mathrm{L}$ of $5 \times$ GC-rich solution, $0.5 \mu \mathrm{L}$ FastStart Taq Polymerase $(5 \mathrm{U} / \mu \mathrm{L})$. For FANCA exons 2-43, the following were added from Invitrogen (Carlsbad, CA): $2 \mu \mathrm{L}$ of $50 \mathrm{mM} \mathrm{MgCl} 2,5 \mu \mathrm{L}$ of $10 \times \mathrm{HiFi}$ buffer, and $0.4 \mu \mathrm{L}$ Platinum Taq HiFi Polymerase $(5 \mathrm{U} / \mu \mathrm{L})$. All PCR amplifications were carried out in a 96-well plate (Eppendorf, Westbury, $\mathrm{NY}$ ) in an Eppendorf thermal cycler (Mastercycler epgradient S). A PCR "touchdown" program was used for the amplification of all 43 exons with the following parameters: initial denaturation at $95^{\circ} \mathrm{C}$ for 4 minutes, followed by amplification of 33 cycles, each with denaturation at $95^{\circ} \mathrm{C}$ for 35 seconds, annealing at $64^{\circ} \mathrm{C}$ for 40 seconds with a decrease of $0.1^{\circ} \mathrm{C} /$ cycle, and extension at $72^{\circ} \mathrm{C}$ for 40 seconds. The final extension at $72^{\circ} \mathrm{C}$ for 5 minutes was followed by a $4^{\circ} \mathrm{C}$ hold step.

FANCA PCR amplification of cDNA, amplicons 2-10 (Table, Supplemental Digital Content 2, http://links.lww.com/A794, FANCA primer sequences; amplicon size; dHPLC parameters ]oven temperature, \% B buffer composition]) was performed as follows: $5 \mu \mathrm{L}$ of the resulting RT-PCR reaction (described 
Table 1 Cytogenetic analysis of FA-A patient fibroblast cells

\begin{tabular}{|c|c|c|c|c|c|c|}
\hline \multirow{2}{*}{$\begin{array}{l}\text { Patient fibroblast } \\
\text { sample }\end{array}$} & \multicolumn{3}{|c|}{$\begin{array}{l}\text { Nontransfected patient cells } \\
\text { Percentage radials (clastogen concentration) }\end{array}$} & \multicolumn{3}{|c|}{$\begin{array}{l}\text { FANCA retroviral transfected patient cells } \\
\text { Percentage radials (clastogen concentration) }\end{array}$} \\
\hline & None, \% (ng/mL) & $\mathrm{MMC}, \%(\mathrm{ng} / \mathrm{mL})$ & DEB, \% (ng/mL) & None, $\%(\mathrm{ng} / \mathrm{mL})$ & MMC, $\%(n g / m L)$ & $\mathrm{DEB}, \%(\mathrm{ng} / \mathrm{mL})$ \\
\hline FA-A1 & $0(0)$ & $42(15)$ & $18(60)$ & $0(0)$ & $0(15)$ & $0(60)$ \\
\hline FA-A2 & $0(0)$ & $52(15)$ & $36(75)$ & $0(0)$ & $0(15)$ & $0(75)$ \\
\hline FA-A3 & $0(0)$ & $43(15)$ & $54(150)$ & $0(0)$ & $2(15)$ & $0(150)$ \\
\hline FA-A4 & $0(0)$ & $30(15)$ & $11(60)$ & $0(0)$ & $0(15)$ & $0(60)$ \\
\hline FA-A5 & $0(0)$ & $64(40)$ & $72(200)$ & $0(0)$ & $6(40)$ & $4(200)$ \\
\hline FA-A6 & $0(0)$ & $78(15)$ & $32(100)$ & $2(0)$ & $0(15)$ & $0(100)$ \\
\hline FA-A7 & $0(0)$ & $57(20)$ & $32(100)$ & $0(0)$ & $14(20)$ & $4(200)$ \\
\hline FA-A8 & $0(0)$ & $56(15)$ & $34(150)$ & $0(0)$ & $0(15)$ & $0(150)$ \\
\hline FA-A9 & $0(0)$ & $13(15)$ & $13(150)$ & $0(0)$ & $1(15)$ & $0(150)$ \\
\hline FA-A10 & $0(0)$ & $36(15)$ & $26(60)$ & $0(0)$ & $0(15)$ & $0(60)$ \\
\hline FA-A11 & $0(0)$ & $40(15)$ & $48(150)$ & $0(0)$ & $2(15)$ & $2(150)$ \\
\hline FA-A12 & $0(0)$ & $52(15)$ & $47(100)$ & $0(0)$ & $0(15)$ & $0(100)$ \\
\hline FA-A13 & $0(0)$ & $36(15)$ & $70(150)$ & $0(0)$ & $0(15)$ & $2(150)$ \\
\hline FA-A14 & $0(0)$ & $44(40)$ & $80(200)$ & $0(0)$ & $8(40)$ & $4(200)$ \\
\hline FA-A15 & $0(0)$ & $50(15)$ & $44(150)$ & $0(0)$ & $6(15)$ & $8(150)$ \\
\hline FA-A16 & $0(0)$ & $38(15)$ & $32(75)$ & $0(0)$ & $2(15)$ & $4(75)$ \\
\hline FA-A17 & $0(0)$ & $38(15)$ & $\mathrm{N} / \mathrm{D}$ & $0(0)$ & $0(15)$ & N/D \\
\hline FA-A18 & $0(0)$ & $32(15)$ & $33(100)$ & $0(0)$ & $0(15)$ & $0(100)$ \\
\hline FA-A19 & $2(0)$ & $15(15)$ & $30(150)$ & $0(0)$ & $0(15)$ & $0(150)$ \\
\hline FA-A20 & $0(0)$ & $28(15)$ & $50(150)$ & $0(0)$ & $2(15)$ & $6(150)$ \\
\hline FA-A21 & $0(0)$ & $8(10)$ & $10(100)$ & $0(0)$ & $0(15)$ & $0(100)$ \\
\hline FA-A22 & $0(0)$ & $50(15)$ & $54(150)$ & $0(0)$ & $0(15)$ & $0(150)$ \\
\hline FA-A23 & $0(0)$ & $46(15)$ & $32(60)$ & $0(0)$ & $0(15)$ & $2.7(60)$ \\
\hline FA-A24 & $0(0)$ & $25(15)$ & $20(60)$ & $0(0)$ & $2(15)$ & $0(60)$ \\
\hline FA-A25 & $0(0)$ & $23(15)$ & $17(150)$ & $0(0)$ & $3(15)$ & $0(150)$ \\
\hline FA-A26 & $4(0)$ & $28(15)$ & $72(150)$ & $0(0)$ & $2(15)$ & $2(150)$ \\
\hline FA-A27 & $0(0)$ & $46(15)$ & $60(150)$ & $0(0)$ & $2(15)$ & $0(150)$ \\
\hline FA-A28 & $0(0)$ & $26(10)$ & $12(100)$ & $0(0)$ & $6(10)$ & $0(100)$ \\
\hline FA-A29 & $0(0)$ & $33(8)$ & N/D & $0(0)$ & $4(8)$ & N/D \\
\hline
\end{tabular}

above) was added to $1 \times \mathrm{HiFi}$ Buffer (Invitrogen), $2 \mathrm{mM}$ $\mathrm{MgSO}_{4}, 0.8 \mathrm{mM}$ each dNTPs, $0.6 \mu \mathrm{M}$ each forward and reverse primers (Table, Supplemental Digital Content 2, http://links.lww.com/A794, FANCA primer sequences; amplicon size; dHPLC parameters [oven temperature, \% B buffer composition]), $0.04 \mathrm{U} / \mu \mathrm{L}$ Platinum HiFi Taq Polymerase (Invitrogen), and water to a total volume of $50 \mu \mathrm{L}$. Amplification cycling conditions were as described for gDNA above except for cDNA amplification, amplicons $2-10,36$ cycles were run and the annealing temperature used was $63^{\circ} \mathrm{C}$. FANCA PCR for amplification of cDNA for amplicon 1 was performed as follows: $5 \mu \mathrm{L}$ of the resulting RT-PCR reaction (described above) was added to $1 \times$ GC-rich solution (Roche), $1 \times$ Roche buffer $+\mathrm{MgCl}_{2}$ (Roche), $0.8 \mathrm{mM}$ each dNTPs, $0.6 \mu \mathrm{M}$ each forward and reverse primers (Table, Supplemental Digital Content 2, http://links.lww.com/A794, FANCA primer sequences; amplicon size; dHPLC parameters [oven temperature, \% B buffer composition]), $0.05 \mathrm{U} / \mu \mathrm{L}$ FastStart Taq polymerase (Roche), and water to a total volume of $50 \mu \mathrm{L}$. Amplification cycling conditions were as described above for gDNA amplification except for cDNA, amplicon 1, 44 cycles were run and the annealing temperature used was $60^{\circ} \mathrm{C}$. Additionally, cell lines 
for which no mutations were detected were sequenced for $F A N C C, G, E, F$ mutations. Primers and cycling conditions for $F A N C C, G, E$, and $F$ sequencing are detailed in our manuscript in preparation. The PCR products were analyzed for quality assurance by running $5 \mu \mathrm{L}$ of the amplified product on $2 \%$ NuSieve (Lonza, Rockland, ME) agarose gel in standard trisacetate buffer at $8 \mathrm{mAmp} / \mathrm{cm}$ gel.

\section{dHPLC analysis}

The PCR products were analyzed for sequence variants by the detection of heteroduplex DNA using dHPLC on the WAVE HT system (Transgenomics, Omaha, NE) using acetonitrile gradient and Navigator software (Transgenomics). For heterozygous mutations, $10-15 \mu \mathrm{L}$ of the amplified PCR product was denatured for 5 minutes. This was followed by a gradual cooling step to $25^{\circ} \mathrm{C}$, at a rate of $1{ }^{\circ} \mathrm{C} /$ minute, to allow for heteroduplex formation. For homozygous mutations, an equimolar amount of patient DNA sample and a negative control were mixed, denatured, and analyzed on dHPLC as described above. Amplicon-specific optimal melting profiles were developed for the FANCA gene (Table, Supplemental Digital Content 2, http://links.lww.com/A794, FANCA primer sequences; amplicon size; dHPLC parameters [oven temperature, \% B buffer composition]) (described in Primer and melting curve profile design section above) and applied to patient samples along with the negative and positive controls. FANCA amplicons were scored as dHPLC positive if a peak shift was detected on the dHPLC chromatogram relative to the appropriate controls.

\section{Sequence analysis}

PCR products from the patient sample scored as dHPLC positive were further analyzed by sequencing. M13 forward and reverse primers were used for all sequencing reactions (Table, Supplemental Digital Content 2, http://links.lww.com/A794, FANCA primer sequences; amplicon size; dHPLC parameters [oven temperature, \% B buffer composition] footnote). All PCR products were sequenced in both forward and reverse directions. Initially, the PCR product was purified using ExoSAP-IT treatment (USB, Cleveland, $\mathrm{OH}$ ) by incubating $5 \mu \mathrm{L}$ of the PCR product with $2 \mu \mathrm{L}$ ExoSAP-IT at $37^{\circ} \mathrm{C}$ for 15 minutes, followed by heat inactivation at $80^{\circ} \mathrm{C}$ for 15 minutes. Half of the incubation mix volume was then aliquoted to a fresh tube. This was followed by adding the M13 forward or reverse primer to the incubation mix to a final concentration of $0.16 \mu \mathrm{M}$. Sequencing reactions were performed using BigDye Terminator v1.1 Cycle Sequencing Ready Reaction-mix kit (Applied Biosystems, Foster City, CA) by adding $1 \mu \mathrm{L}$ of the BigDye Terminator and 1.5 $\mu \mathrm{L}$ of $5 \times$ BigDye buffer in a final sequencing reaction volume of $10 \mu \mathrm{L}$. PCR sequencing thermal cycling profile were: initial step of $96^{\circ} \mathrm{C}$ for 1 minute; 25 cycles at $96^{\circ} \mathrm{C}$ for 10 seconds, $50^{\circ} \mathrm{C}$ for 5 seconds, $60^{\circ} \mathrm{C}$ for 4 minutes. Sequence extension products were purified using DyeEx 96 gel filtration plate (Qiagen, Valencia, CA) in accordance to manufacturer's instructions. The purified sequencing products were transferred to an Optical 96-well plate (Applied Biosystems) and dried in a $70^{\circ} \mathrm{C}$ heat block with forced ventilation for 15 minutes. The sequencing products were resuspended in $15 \mu \mathrm{L}$ of Hi-Di formamide (Applied Biosystems) and separated on ABI PRISM 3100 Genetic Analyzer using a 50-cm capillary array and POP6 polymer (Applied Biosystems). DNA trace sequences were generated from raw sequence data using Sequencing Analysis v5.1.1 (Applied Biosystems) and analyzed for sequence information using Mutation Surveyor software, version 3.01 (Soft Genetics, State College, PA). Novel variants detected were analyzed in silico as described in Results section; functional complementation assays were not performed.

\section{MLPA analysis}

Multiplex ligation-dependent probe amplification (MLPA) analysis was performed to determine relative copy numbers of all $43 F A N C A$ exons in all the mutation-negative, heterozygousand homozygous-mutation patient samples. An MLPA kit for the FANCA gene, SALSA P031/P032, was used in this study (MRC-Holland, Amsterdam, Netherlands). Briefly, $200 \mathrm{ng}$ of gDNA was denatured and hybridized with each of SALSA P031 and $\mathrm{P} 032$ probes following the manufacturer's instructions. This was followed by ligation and PCR amplification according to manufacturer's instructions. One microliter of the probe amplification product was then mixed with $0.3 \mu \mathrm{L}$ of the GeneScan500 ROX size standard (Applied Biosystems), $9 \mu \mathrm{L}$ of HiDi formamide (Applied Biosystems) and resolved using the ABI 3100 Avant Genetic Analyzer with a 36-cm capillary array and POP4 polymer (Applied Biosystems). GeneMapper v.3.5 software (Applied Biosystems) was used for fragment analysis and the resulting peak height and size area were exported to Excel files. All subsequent quantitative and statistical analysis of the data were performed using FANCA Excel templates. In short, the peak heights (and areas) of the expected MLPA products were normalized by dividing each peak height (and area) by the combined height (and area) of all peaks in the sample. For the three normal controls, the mean $(n=3)$ of the normalized peak heights (and areas) was calculated from all the probes for each probe-mix P031 and P032. For the patient sample and the positive control, each of the normalized peak height (and area) was divided by the corresponding mean of the normalized peak height (and area) of the normal controls $(n=3)$. The resulting value for each FANCA exon was compared with those of internal controls of nearest neighbor. All exons differing by more than $30 \%$ from the normalized peak heights (and areas) of internal controls of comparable fragment size were considered as altered; reduced values indicated a deletion, whereas increased values indicated a duplication.

\section{RESULTS}

\section{Validation of complementation group assignment to FA-A}

FA clinical diagnoses were confirmed and FA-A complementation group assignments were made as described above (see Materials and Methods) for 29 patient-derived cell lines. gDNAs extracted from these fibroblasts were molecularly tested as shown in Figure 1. All samples were rapidly scanned by dHPLC for heterozygous mutations and assayed for gross deletions by MLPA. Samples that were positive by dHPLC were analyzed by direct sequencing. Those samples which lacked two detectable mutations were further screened for homozygous mutations using dHPLC, i.e., mixing patient DNA with a DNA from a negative control. The remainder of the FA samples which showed no or only one mutation were analyzed by direct sequencing of the entire gene and, for the purpose of this study, followed by cDNA analysis. After these analyses, if no mutation was detected in a sample, full sequencing of FANCC, $G, E$, and $F$ was performed.

Of the 29 FA-A assigned patients, 25 were either homozygous or compound heterozygous for putative pathogenic mutations in FANCA (Table 3). The possibility of allele dropout was excluded for four of the five homozygous samples through the 


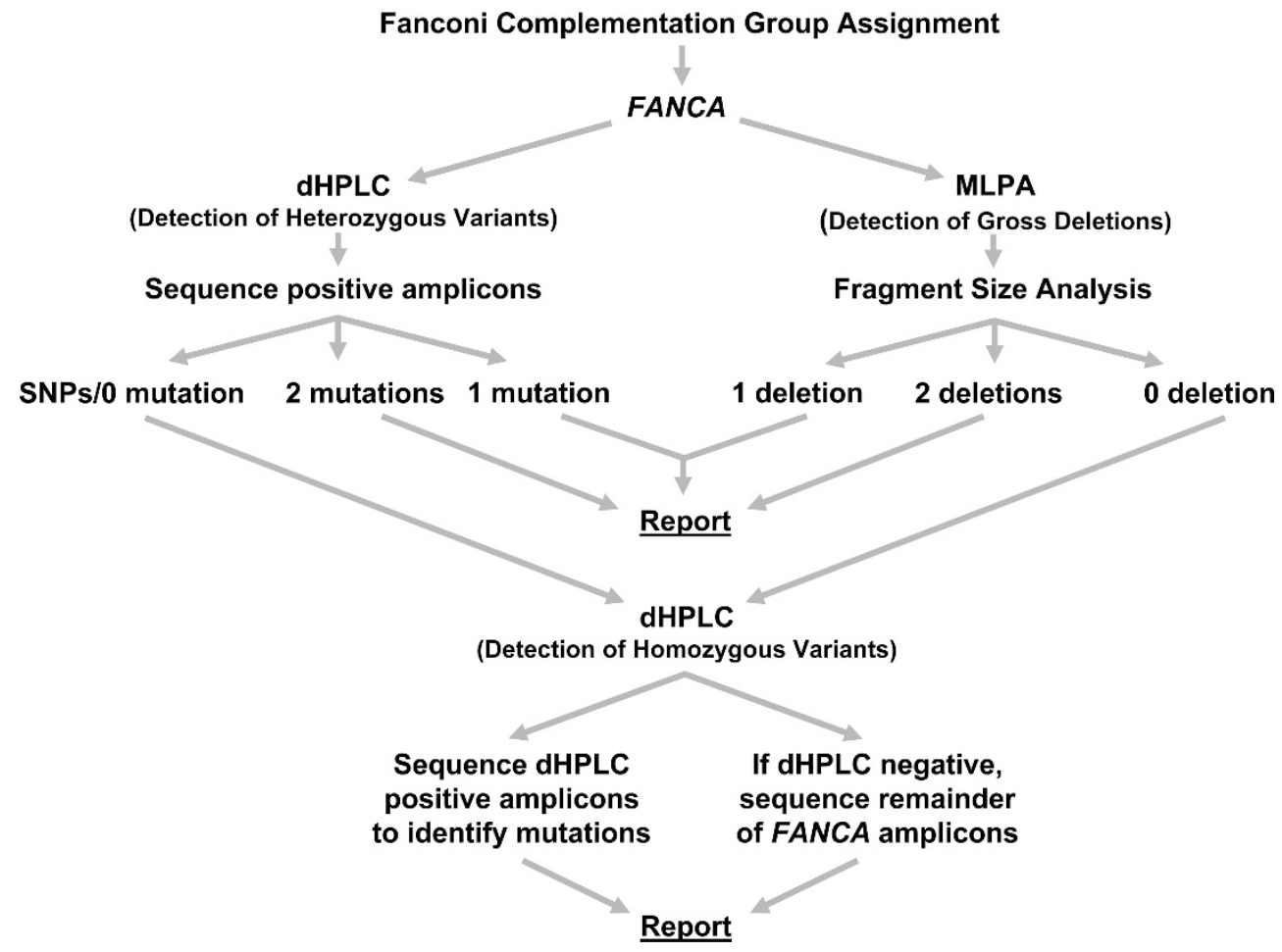

Fig. 1. FANCA gene analysis scheme for the clinical molecular diagnosis of FA-A patients. FA patients were initially assigned to a specific complementation group by cytogenetic analysis of fibroblast cells. "Mutation" signifies pathogenic variant. dHPLC, denaturing high pressure liquid chromatography; MLPA, multiplex ligation-dependent probe amplification; SNP, single nucleotide polymorphism.

use of alternate primer sets. In the fifth sample (FA-A12), a homozygous gross deletion was detected by MLPA, dHPLC, and by sequencing thus minimizing the likelihood of the homozygosity resulting from a SNP in a probe/primer site. Of the remaining four FA-A assigned samples, two of the samples revealed a single heterozygous mutation (one known and one putative pathogenic mutation), whereas no discernable mutation was detected in the remaining two samples.

A total of 31 different known pathogenic mutations and eight different novel putative pathogenic mutations were identified in this study. The pathogenicity of the novel variants was assessed through interrogation by splice site algorithms (intronic and exonic) and by protein structure and function algorithms. ${ }^{63-68}$
The novel mutations detected were strongly suggested by algorithms to be pathogenic, although they were not tested by functional complementation analysis; one of these novel mutations occurred at an obligatory donor splice site, four were nonsense, one was missense, one was a gross duplication which by cDNA had a multiexon skip embedded in the duplication region, and one was a 138 bp deletion (Table 3).

A schematic representation of FANCA and the pathogenic mutations reported in this study are shown in Figure 2. Four novel sequence variants, not shown on Figure 2, (c.4167+ $46 \mathrm{C}>\mathrm{T} ;$ c. $893+71 \mathrm{~T}>\mathrm{C} ;$ c. $2317-49 \mathrm{C}>\mathrm{G} ;$ c.3349-47C $>\mathrm{G})$ were detected and are tentatively being designated as nonpathogenic based on analysis by splice site prediction algo-

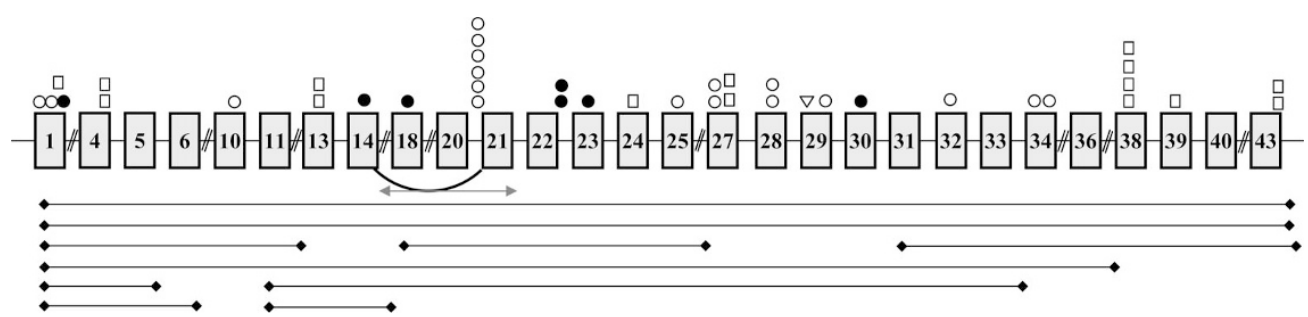

Fig. 2. FANCA gene structure and distribution of mutations. Schematic representation of FANCA gene structure with the relevant exon number shown (shaded boxes). Location of mutations, excluding gross deletions, reported in this study is shown above the diagram, whereas those for gross deletions are shown below the diagram. Identical mutations are indicated by symbols lining up vertically. Gaps between exons are indicated by "//." Schematic is not drawn to scale. 0 , novel point mutation; $\bigcirc$, known point mutation; $\square$, known small deletion; $\nabla$, known insertion; $\bullet$, known gross deletion; $\longrightarrow$, novel exon skipping; $\leftrightarrow$, novel gross duplication. 


\begin{tabular}{|c|c|c|c|c|c|c|c|}
\hline Patient number & FA-A3 & FA-A9 & FA-A19 & FA-A25 & FA-A26 & FA-A27 & FA-A28 \\
\hline Age & $12 \mathrm{yr}$ & $12 \mathrm{yr}$ & $24 \mathrm{yr}$ & $24 \mathrm{yr}$ & $15 \mathrm{yr}$ & $1 \mathrm{mo}, 22$ days & $7 \mathrm{yr}$ \\
\hline Gender & $\mathrm{F}$ & M & $\mathrm{F}$ & $\mathrm{F}$ & F & $\mathrm{F}$ & $\mathrm{F}$ \\
\hline Mutant allele 1 & c. $1827-1 \mathrm{G}>\mathrm{A}$ & c.3391A > G [p.T1131A] & c. $2524 \mathrm{delT}$ & c. $2524 \mathrm{delT}$ & c. $1827-1 \mathrm{G}>\mathrm{A}$ & DelExon 1-6 & None detected \\
\hline Mutant allele 2 & c.3920delA & DelExon 11-33 & c. $2639 \mathrm{G}>\mathrm{A}[\mathrm{p} . \mathrm{R} 880 \mathrm{Q}]$ & c. $2639 \mathrm{G}>\mathrm{A}$ [p.R880Q] & c.3349A>G [p.R1117G] & DelExon 11-14 & None detected \\
\hline Ethnicity & White & White & White & White & Unknown & White & Hispanic \\
\hline Consanguinity & No & No & No & No & Yes (distant) & No & No \\
\hline $\begin{array}{l}\text { Blood-low red } \\
\text { blood cell }\end{array}$ & Yes & Yes & No & No & Yes & No & Yes (since age 5) \\
\hline $\begin{array}{l}\text { Low white } \\
\text { blood cell }\end{array}$ & Yes & Yes & No & No & No & No & Yes \\
\hline $\begin{array}{r}\text { Low blood } \\
\text { platelets }\end{array}$ & Yes & Yes & No & No & Yes & No & Yes \\
\hline Bleeding & No & No & No & No & Yes & No & No \\
\hline $\begin{array}{l}\text { Bone marrow } \\
\text { transplantation }\end{array}$ & No & No & No & No & No & No & No \\
\hline $\begin{array}{l}\text { Serious } \\
\quad \text { infection }\end{array}$ & No & No & No & No & No & No & No \\
\hline $\begin{array}{l}\text { Absent or } \\
\text { malformed } \\
\text { thumb }\end{array}$ & $\begin{array}{l}\text { Extra thumb at } \\
\text { right hand }\end{array}$ & Absent/malformed thumb & Absent thumb & Hypoplastic thumb & Malformed thumb & Absent thumb & $\begin{array}{l}\text { Absent/malformed } \\
\text { thumb }\end{array}$ \\
\hline $\begin{array}{l}\text { Other skeletal } \\
\text { anomalies }\end{array}$ & $\begin{array}{l}\text { Partial fusion of } \\
\text { heel joints; flat } \\
\text { feet }\end{array}$ & No & Fused vertebrae in neck & No & $\begin{array}{l}\text { Hypoplastic radius; bone } \\
\text { pain }\end{array}$ & No & No \\
\hline $\begin{array}{l}\text { Short stature or } \\
\text { delayed } \\
\text { growth }\end{array}$ & No & Yes & $\begin{array}{l}\text { Yes (below zero } \\
\text { percentile) }\end{array}$ & $\begin{array}{l}\text { Yes (below zero } \\
\text { percentile) }\end{array}$ & Yes & Yes & Yes \\
\hline $\begin{array}{l}\text { Skin } \\
\quad \text { pigmentation }\end{array}$ & $\begin{array}{l}\text { Two café au lait } \\
\text { spots on thigh } \\
\text { (at age 8) }\end{array}$ & Yes (at age 12) & No & Yes (at infancy) & No & No & Yes \\
\hline $\begin{array}{l}\text { Kidney or } \\
\text { urinary } \\
\text { problems }\end{array}$ & No & No & No & Yes & No & $\begin{array}{l}\text { Missing (left) } \\
\text { kidney }\end{array}$ & Yes \\
\hline $\begin{array}{l}\text { Leukemia or } \\
\text { other cancer }\end{array}$ & No & No & No & No & No & No & No \\
\hline $\begin{array}{l}\text { Other health } \\
\text { issues }\end{array}$ & No & No & No & No & Schizoaffective disorder & Hypothyroidism & No \\
\hline $\begin{array}{l}\text { Learning } \\
\quad \text { difficulties }\end{array}$ & No & No & No & No & Yes & No & No \\
\hline
\end{tabular}

rithms. ${ }^{63-66}$ Also not shown in Figure 2 are three samples (FA-A18, FA-A28, FA-A29) determined to be heterozygous for exon 30 skipping; this skipping has been reported to be benign. 44

Thus, complementation assignment to FA-A was molecularly confirmed in 25 cell lines, strongly suggested in two additional cells, and unconfirmed in two cells lines; the unconfirmed FA-A cell lines are discussed further in the Discussion section.

\section{Clinical phenotypic data}

Table 2 shows available clinical and genotype information for 7 of 29 patients studied. All seven patients were assessed on the basis of three primary clinical features associated with the FA: progressive pancytopenia, physical abnormalities, and cancer susceptibility. All patients had radial ray defects, all but one displayed delayed growth, and most (excluding the monozygotic twins who exhibited reversion and are discussed below) evidenced pancytopenia. Other physical abnormalities displayed by some included skin pigmentation, kidney disease, skeletal anomalies, and learning difficulties. None of our patients exhibited leukemia or other cancers at the time of diagnosis (Table 2). No genotype/phenotype correlation is evident in this small cohort.

Two of our patients are monozygotic twin sisters (FA-A19, FA-A25) who were clinically evaluated at ages 13 years ${ }^{69}$ and 28 years. ${ }^{70}$ At the time of evaluation, both twins were free from any hematologic signs and were found to be mosaic because of somatic reversion at the level of hematopoietic stem cells. ${ }^{69}$ Neither twin had any sign of hematologic symptoms such as myeloid leukemia and myelodysplasia, or solid tumors by age 28 (Table 2). 


\section{Analytical test validation-specificity and sensitivity}

The coding region and splice junctions of FANCA were analyzed in four negative control gDNA samples as shown in Figure 1. For two of these controls, no deletions/duplications or variants were detected as determined by comparison with the FANCA reference sequence (GenBank NT_010542.15). Numerous variants, identified as known polymorphisms, were detected in the other two negative controls (data not shown). As no FANCA pathogenic mutations were detected in the negative control samples, the resulting analytical test specificity was determined to be $100 \%$. The negative controls lacking SNPs were used in subsequent tests for dHPLC and sequence analysis.

Eighteen FANCA mutant alleles having known point mutations or small deletions were PCR amplified in the region of the gene carrying the mutation and analyzed by dHPLC and sequencing (Table, Supplemental Digital Content 1, http://links.lww.com/A777, Positive controls used for FANCA test development). Each of the 18 mutant alleles showed a peak shift on their respective WAVE chromatogram relative to the negative control; all were subsequently sequenced resulting in verification of the presence of each mutation. In addition, a FANCA mutant allele with a known gross deletion was verified by MLPA analysis (Table, Supplemental Digital Content 1, http://links.lww.com/A777, Positive controls used for $F A N C A$ test development). Thus, the analytical sensitivity for this sample set was $100 \%$.

\section{Clinical test validation-sensitivity and specificity}

The mutation detection rate of FANCA for our cohort of 29 FA-A identified patient-derived cell lines was $89 \%$, i.e., 50 of 56 presumed $F A N C A$ mutations were detected (the alleles from one of the identical twins of the pair in this study was excluded for this calculation) (Table 3$)$. Of these, $80 \%(n=40)$ were heterozygous pathogenic mutations; $20 \%(n=10)$ were homozygous pathogenic mutations. Nine of the mutations were missense; seven were nonsense mutations; eight were splice site mutations; eight were small insertions/deletions resulting in a frame shift; five were deletions resulting in an amino acid deletion; 10 were multiexon gross deletions; two were deletions of $138 \mathrm{bp}$; and one was exon skipping embedded in a gross duplication. Novel (unreported) mutations $(n=10)$ are shown in Table 3. The pathogenicity of novel mutations was determined as described above. Two patient samples will be discussed below in which no mutations were detected.

The clinical sensitivity of the molecular analysis is estimated at $86 \%$ with two pathogenic $F A N C A$ mutations identified in 24 of 28 putative FA-A patients. All mutations, excluding the gross deletions ( $20 \%$ of total mutations detected) and gross duplication, were detected by dHPLC and confirmed by sequencing. Thirteen patient samples were fully sequenced ( 26 alleles); none of these yielded a mutation by sequencing that was undetected by dHPLC.

No pathogenic FANCA mutations were detected in the negative control samples, thus the resulting clinical and analytical test specificity was determined to be $100 \%$.

\section{DISCUSSION}

Because of the high degree of clinical variability exhibited by FA patients and the phenotypic overlap with other radial ray defect disorders such as Diamond-Blackfan Anemia (characterized by anemia and abnormal thumbs) and to a lesser degree TAR (anemia and thrombocytopenia-absent radius syndrome), as well as pancytopenia phenotypic overlap with dyskeratosis congenita, confirmation of FA is routinely performed cytogenetically; peripheral blood and/or fibroblasts are analyzed for hypersensitivity to MMC and DEB, i.e., evidenced by a high rate of chromosomal breakage and radial formation. The 29 patient-derived cell lines studied here were referred for testing based on clinical phenotypes and were subsequently confirmed cytogenetically to be positive for FA.

Because of the genetic heterogeneity of FA, with at least 13 genes being implicated, efficient molecular testing is challenging in a clinical setting. Except for the recently reported approach of Ameziane et al., ${ }^{47}$ the reported testing strategies, including ours, rely on complementation group analysis to enable efficient molecular testing. In this study, we make a novel contribution to the literature by describing FANCA complementation group assignment validation through unique comprehensive molecular verification of FA-A assignments (based on retroviral correction of chromosome breakage and radial formation) in a clinical setting.

All 29 of our patient-derived fibroblasts exhibited normal breakage and radial results after $F A N C A$-loaded retroviral transfection resulting in assignment to FA complementation Group A (FA-A); FA-A is the largest FA subtype representing $66 \%$ of FA patients.

Validation of complementation assignment required that we develop a rapid, efficient, and comprehensive molecular mutation detection strategy for FANCA. To facilitate capture of all mutations, our analysis strategy integrated rapid mutation scanning (dHPLC), deletion/duplication testing (MLPA), and reflex direct sequencing. As a result of this study, we recently began offering full FANCA clinical mutation testing as provider for this service in the United States (GeneTests).

For efficient molecular mutation screening, we designed 42 sets of M13-tailed primer pairs for simultaneous robust PCR amplification, optimal dHPLC analysis and efficient sequencing of the entire coding region and flanking sequences of $F A N C A$. Because $F A N C A$ is highly polymorphic, extra care was taken during primer design to avoid SNPs in an effort to ensure consistent biallelic amplification of the gene in patient DNA. Additionally, we integrated MLPA into the testing strategy to facilitate the capture of gross deletions and duplications.

We validated our test for analytical specificity by evaluating $F A N C A$ in gDNA samples from four apparently normal and unrelated individuals. Using dHPLC, MLPA, and full gene sequencing of these samples, no false positives were detected. Analytical sensitivity was assessed using FANCA positive control samples. All mutations in the collection of control samples (Table, Supplemental Digital Content 1, http://links.lww.com/A777, Positive controls used for FANCA test development) were detected and identified correctly.

The clinical sensitivity was determined by molecular testing of our FA-A assigned patient cohort. Of the 28 FA-A assigned patient samples ( 56 mutant alleles, see footnote in Table 3 for explanation), two putative pathogenic FANCA mutations were detected in 24 patients ( 48 mutant alleles), confirming the FA-A complementation assignment; a single presumed $F A N C A$ pathogenic mutation was detected in two patients (two mutant alleles), strongly supporting FA-A subtype; and no discernable FANCA mutation was detected in the remaining two patient samples. The 50 putative pathogenic mutant alleles detected in this cohort correspond to an $89 \%$ mutation detection rate (based on alleles), and an $86 \%$ clinical sensitivity (based on genotype) which compare favorably with the $83 \%$ clinical sensitivity previously published. ${ }^{55}$

Eight mutations detected in this study were unreported. The pathogenicity of these novel variants was assessed by splice site 
Table 3 FANCA gene mutations and deletions detected in FA-A patients

\begin{tabular}{|c|c|c|c|}
\hline Sample & Genotype & Mutation type & References \\
\hline FA-A1 & c. $1827-1 \mathrm{G}>\mathrm{A} / \mathrm{c} .1827-1 \mathrm{G}>\mathrm{A}$ & Acceptor splice site/acceptor splice site & $62 / 62$ \\
\hline FA-A2 & c. $1827-1 \mathrm{G}>\mathrm{A} / \mathrm{delExon} 1-5$ & Acceptor splice site/gross deletion & $62 / 55$ \\
\hline FA-A3 & c. $1827-1 \mathrm{G}>\mathrm{A} / \mathrm{c} .3920 \mathrm{del} \mathrm{A}$ & Acceptor splice site/1bp deletion (frameshift) & $62 / 35$ \\
\hline FA-A4 & c. $2014+1 \mathrm{G}>\mathrm{C} / \mathrm{c} .2014+1 \mathrm{G}>\mathrm{C}$ & Donor splice site/donor splice site & Novel/novel \\
\hline FA-A5 & delExon 1-11/dupExons 15-21 & Gross deletion/exon ${ }^{15-20}$ skipping & $62 /$ novel \\
\hline FA-A6 & c. $1645 \mathrm{C}>\mathrm{T} / \mathrm{c} .2831 \mathrm{dup} 2812-2830$ & p.Q549X (nonsense)/19 bp insertion (frameshift) & Novel/46 \\
\hline FA-A7 & c. $2854 \mathrm{C}>\mathrm{T} / \mathrm{c} .1304 \mathrm{G}>\mathrm{A}$ & p.Q952X (nonsense)/R435H (missense) & Novel/novel \\
\hline FA-A8 & c. $2 \mathrm{~T}>\mathrm{C} /$ delExon $1-36$ & p.M1T (missense)/gross deletion & $62 / 62$ \\
\hline FA-A9 & c. $3391 \mathrm{~A}>\mathrm{G} /$ delExon $11-33$ & p.T1131A (missense)/gross deletion & $47 / 47$ \\
\hline FA-A10 & c.65G $>$ A/c.3788_3790delTCT & p.W22X (nonsense)/p.F1263del (deletion) & $62 / 35$ \\
\hline FA-A11 & c. $3163 \mathrm{C}>\mathrm{T} /$ delExon $18-25$ & p.R1055W (missense)/gross deletion & $35 / 62$ \\
\hline FA-A12 & c.4268_4368+37del138/c.4268_4368+37del138 & Gross deletion/gross deletion & Novel/novel \\
\hline FA-A13 & c.44_69del26/delExon 1-43 & 26bp deletion (frameshift)/gross deletion & $62 / 31$ \\
\hline FA-A14 & c.1115_1118delTTGG/c.2534T >C & 4 bp deletion (frameshift)/p.L845P (missense) & $35 / 53$ \\
\hline FA-A15 & c. $13 \mathrm{G}>\mathrm{A} /$ delExon $31-43$ & p.W5X (nonsense)/gross deletion & Novel/62 \\
\hline FA-A16 & c.3788_3790delTCT/c.3788_3790delTCT & p.F1263del (deletion)/p.F1263del (deletion) & $35 / 35$ \\
\hline FA-A17 & c.416_417delTG/c.416_417delTG & $2 \mathrm{bp}$ deletion (frameshift)/2bp deletion (frameshift) & $62 / 62$ \\
\hline FA-A18 & c. $2021 \mathrm{C}>\mathrm{A} /$ undetected & p.S674X (nonsense)/unknown & Novel \\
\hline $\mathrm{FA}-\mathrm{A} 19^{a}$ & c. $2524 \mathrm{delT} / \mathrm{c} .2639 \mathrm{G}>\mathrm{A}$ & $1 \mathrm{bp}$ deletion (frameshift)/p.R880Q (missense) & $62 / 62$ \\
\hline FA-A20 & delExon 1-43/undetected & Gross deletion/unknown & 31 \\
\hline FA-A21 & c. $862 \mathrm{G}>\mathrm{T} / \mathrm{c} .1115 \_1118 \mathrm{delTTGG}$ & p.E288X (nonsense)/4bp deletion (frameshift) & $55 / 50$ \\
\hline FA-A22 & c. $2303 \mathrm{~T}>\mathrm{C} / \mathrm{c} .2534 \mathrm{~T}>\mathrm{C}$ & p.L768P (missense)/p.L845P (missense) & $62 / 52$ \\
\hline FA-A23 & c.2167_2169delCTG/c.3788_3790delTCT & p.L723del (deletion)/p.F1263del (deletion) & $35 / 35$ \\
\hline FA-A24 & c. $1827-1 \mathrm{G}>\mathrm{A} / \mathrm{c} .2840 \mathrm{C}>\mathrm{G}$ & Acceptor splice site/p.S947X (nonsense) & $62 / 46$ \\
\hline $\mathrm{FA}-\mathrm{A} 25^{a}$ & c. $2524 \mathrm{delT} / \mathrm{c} .2639 \mathrm{G}>\mathrm{A}$ & $1 \mathrm{bp}$ deletion (frameshift)/p.R880Q (missense) & $62 / 62$ \\
\hline FA-A26 & c. $1827-1 \mathrm{G}>\mathrm{A} / \mathrm{c} .3349 \mathrm{~A}>\mathrm{G}$ & Acceptor splice site/p.R1117G (missense) & $62 / 31$ \\
\hline FA-A27 & delExon 1-6/delExon 11-14 & Gross deletion/gross deletion & $62 / 35$ \\
\hline FA-A $28^{b}$ & No mutation detected & No mutation detected & \\
\hline $\mathrm{FA}-\mathrm{A} 29^{b}$ & No mutation detected & No mutation detected & \\
\hline
\end{tabular}

${ }^{a}$ Following completion of the complementation validation study, the samples were unblinded. Our molecular results agreed with previously published results for these twins ${ }^{69,70}$ Because FA-A19 and FA-A25 are identical twins, only one set of alleles was counted in the assay validation analysis.

${ }^{b}$ FA-A28 and FA-A29 could not be confirmed by molecular analysis to be in FA-A complementation group. Thus, these four alleles are considered putative FANCA alleles. See Discussion section.

mutation algorithms (intronic and exonic) and by protein structure and function algorithms. ${ }^{63-68}$ One of these novel mutations occurred at an obligatory donor site; four were nonsense; one was missense, one was a 138 bp deletion, and one allele (FAA5) had a gross duplication (Table 3). ${ }^{55}$ Upon further cDNA study of this final sample, a multiple-exon skipping that disrupts the reading frame was detected. Gualandi et al. ${ }^{71}$ recently reported similar cases of exon skipping occurring within duplicated regions. Although the missense mutation is of less certainty as to pathogenicity and could be further analyzed using an in vitro functional assay, it was found along with a nonsense mutation in a symptomatic patient for which break- age analysis confirmed FA and complementation analysis indicated FA-A.

The majority, $78 \%$, of the mutations identified in this study ( $n=39)$ were detected by dHPLC and confirmed by direct sequencing. These include point mutations, small deletions, and a duplication (Table 3). Full gene sequencing was performed on 13 gDNA samples; all alleles that were negative by dHPLC were also negative by sequencing. Ten of the mutant alleles had a gross deletion for the $F A N C A$ gene, corresponding to $20 \%$ of the 50 mutant alleles detected, which is significantly lower than the $40 \%(n=26)$ reported elsewhere. ${ }^{55}$ The four patient samples for which only one or no mutations were detected were 
further studied by cDNA analysis. We found three of these four cell lines to be heterozygous for skipping exon 30. Exon 30 skipping maintains the reading frame and has been previously reported as benign. ${ }^{44}$

Clinical specificity is of special interest in that the FANCA gene is known to be highly polymorphic with approximately 1300 SNPs being reported thus far. ${ }^{60}$ By reflexing all dHPLC positive wave shifts to sequencing, the specificity of this assay was $100 \%$.

Thus, as evidenced by this study, FA-A complementation group assignment did, in the vast majority of instances (26 of 28 ), result in correct identification of the mutated FA gene of interest thereby enabling efficient FA molecular testing. Considering the two cell lines for which only one pathogenic mutation was detected, we suggest the second mutation might be located in cis- or in trans-acting regulatory regions of the $F A N C A$ gene that include the promoter region and enhancer elements for which our assay does not detect. These molecular results of two symptomatic patients having only one identifiable gene mutation do, in fact, support the diagnosis of FA.

Of more concern are the two patient cell lines for which no mutations were found by dHPLC, by direct sequencing of the entire FANCA gene, by MLPA, or by cDNA analysis. Several explanations are possible. The possibility that pathogenic mutations within the FANCA gene were missed cannot be excluded as our current analysis does not detect mutations in regulatory regions. It is also possible that a mutation in the promoter could lead to a reduced transcript or an altered tissue-specific transcript. Because we tested fibroblasts rather than lymphocytes, reversion would not be a likely explanation for the paucity of expected mutations in this subset of patient samples. Another possibility is misassignment of these two patients to complementation group FA-A on the basis of artifacts caused by retroviral transfection and/or tissue culture. Assignment to FA-A could be incorrect because of overexpression of the FANCA protein in the complemented cells. To evaluate this possibility, we have examined these two cell lines for mutations in FANCC, FANCG, and FANCF, and one of these cell lines for $F A N C E$ as well, and have not identified a pathogenic mutation. It is possible that these patients could be in a new complementation group which is yet undefined. In addition, we presently cannot rule out a preanalytical, analytical, or postanalytical error in the complementation assay. We have performed complete molecular analyses in our Clinical Laboratory Improvement Amendment 1988 (CLIA)-approved/College of American Pathologists-certified clinical laboratory where we routinely perform other rare disease testing using similar methods. The complementation analysis was performed in a research setting. If we assume that the two patient specimens in which no mutations were identified are not FA-A because of an error in complementation assignment, the accuracy of complementation analysis would drop from $28 / 28$, or $100 \%$, to $26 / 28$, or $93 \%$. Given that complementation group assignment is really used as a screening tool before molecular analysis, a 93\% detection rate would not be too unreasonable compared with other types of medical screening, such as carrier screening for cystic fibrosis. However, it should be noted that several independent studies have likewise found only one or no mutations identified in a minority of their FA patient population ${ }^{31,35,43,47}$ suggesting that our findings are well within reason. If these two cell lines are in fact not FA-A, then the clinical sensitivity of our molecular analysis would be $96 \%$ based on allele detection rate, and based on genotype, $93 \%$ for two mutations identified and $100 \%$ for at least one mutation identified.
Although complementation assignment has been shown in this study to be a valuable tool enabling clinically feasible molecular analysis, the two unconfirmed FA subtype cell lines underscore the companion need to use molecular testing to confirm complementation group assignment. In addition, molecular testing for FA is an invaluable tool for patient care as it is unique in providing a means to perform the following: (1) identification of carriers (not possible by breakage/radial analysis); (2) prenatal testing of familial mutations (breakage/radial assessment is difficult to perform because of lack of prenatal controls); (3) assessment of familial mutations in family members of affected individuals; (4) determination of mode of inheritance (autosomal or X-linked); (5) resolution of ambiguous cytogenetic testing; (6) verification of bone marrow donor, i.e., exclusion of presymptomatic FA donors; (7) preimplantation genetic diagnosis; (8) aid in clinical management, i.e., enables use of genotype-phenotype published data; and (9) diagnostic exclusion of overlapping chromosome fragility diseases, e.g., Nijmegen Breakage Syndrome, Ataxia-Telangiectasia, and Bloom Syndrome.

In summary, we found our integrated molecular testing strategy using dHPLC, MLPA, and direct sequencing to be efficient, sensitive, and specific for the detection of FANCA mutations in a clinical laboratory setting. Using this testing strategy, we have validated retroviral transfection-mediated complementation assignment for FA-A for clinical usage. Additionally, we identified several novel pathogenic mutations. Finally, we suggest that molecular testing of FA patients provides information integral to best knowledge-based patient management.

\section{ACKNOWLEDGMENTS}

The authors thank The Fanconi Anemia Research Fund (FARF) for support of Grant titled "Development of a Comprehensive Clinical Program for Fanconi Anemia Diagnosis: A Molecular Approach" (2006-2008); Yassmine Akkari and Amy Hanlon Newell at Oregon Health \& Science University for providing information on the FA-A assigned complement cell lines; Akira Tachibana, Ibaraki University, Ibaraki, Japan; Maria Castella and Jordi Surralles (manuscript in preparation), Universidad Autonoma de Barcelona, Barcelona, Spain; Christopher G. Mathew, King's College London School of Medicine, London, UK and Lisa Barber, St. Mary's Hospital, Manchester, UK for providing the positive control genomic DNA.

\section{REFERENCES}

1. Verlander PC, Kaporis A, Liu Q, Zhang Q, Seligsohn U, Auerbach AD. Carrier frequency of the IVS4 $+4 \mathrm{~A}->\mathrm{T}$ mutation of the Fanconi anemia gene FAC in the Ashkenazi Jewish population. Blood 1995;86:4034-4038.

2. Callén E, Casado JA, Tischkowitz MD, et al. A common founder mutation in FANCA underlies the world's highest prevalence of Fanconi anemia in Gypsy families from Spain. Blood 2005;105:1946-1949.

3. Alter BP. Fanconi's anemia and malignancies. Am J Hematol 1996;53:99-110.

4. Akkari YMN, Olson SB. Fanconi Anemia: a decade of discoveries. J Assoc Genet Technol 2004;30:48-53.

5. Fanconi G. Familial constitutional panmyelocytopathy, Fanconi's anemia (FA). I. Clinical aspects. Semin Hematol 1967;4:233-240.

6. Glanz A, Fraser FCJ. Spectrum of anomalies in Fanconi anemia. Med Genet 1982;19:412-416.

7. Auerbach AD, Allen RG. Leukemia and preleukemia in Fanconi anemia patients: a review of the literature and report of the international Fanconi anemia registry. Cancer Genet Cytogenet 1991;51:1-12.

8. Tischkowitz M, Dokal I. Fanconi anemia and leukemia. Clin Mol Aspects 2004;126:176-191

9. Auerbach AD. Fanconi anemia diagnosis and the diepoxybutane (DEB) test. Exp Hematol 1993;21:731-733.

10. Shimamura A, Montes de Oca R, Svenson JL, et al. A novel diagnostic screen for defects in the Fanconi anemia pathway. Blood 2002;100:46494654.

11. Ponk JC, Gibson RA, Savoia A, Wijker M, et al. Localization of the Fanconi 
anaemia complementation group A gene to chromosome 16q24.3. Nat Genet 1995; 11:338-340.

12. Loe Ten Foe JR, Rooimans MA, Bosnoyan-Collins L, et al. Expression cloning of a cDNA for the major Fanconi anaemia gene, FAA. Nat Genet 1996; 14:320-323.

13. Meetei AR, Levitus M, Xue Y, et al. X-linked inheritance of Fanconi anemia complementation group B. Nat Genet 2004;36:1219-1224.

14. Strathdee CA, Gavish H, Shannon WR, Buchwald M. Cloning of cDNAs for Fanconi's anaemia by functional complementation. Nature 1992;358:434.

15. Howlett NG, Taniguchi T, Olson S, et al. Biallelic inactivation of BRCA2 in Fanconi anemia. Science 2002;297:534

16. Timmers $\mathrm{C}$, Taniguchi T, Hejna J, et al. Positional cloning of a novel Fanconi anemia gene, FANCD2. Mol Cell 2001;7:241-248.

17. DeWinter JP, Rooimans MA, VanDerWeel L, et al. The Fanconi anaemia gene FANCF encodes a novel protein with homology to ROM. Nat Genet $2000 ; 24: 15-16$

18. Dorsman JC, Levitus M, Rockx D, et al. Identification of the Fanconi anemia complementation group I gene, FANCI. Cell Oncol 2007;29:181-182.

19. Sims AE, Spiteri E, Sims RJ 3rd, et al. FANCI is a second monoubiquitinated member of the Fanconi anemia pathway. Nat Struct Mol Biol 2007;14:564567.

20. Smogorzewska A, Matsuoka S, Vinciguerra $\mathrm{P}$, et al Identification of the FANCI protein, a monoubiquitinated FANCD2 paralog required for DNA repair. Cell 2007;129:289-301

21. Levitus M, Waisfisz Q, Godthelp BC, et al. The DNA helicase BRIP1 is defective in Fanconi anemia complementation group. J Nat Genet 2005;37: 934-935

22. Levran O, Attwooll C, Henry RT, et al. The BRCA1-interacting helicase BRIP1 is deficient in Fanconi anemia. Nat Genet 2005;37:931-933.

23. Litman R, Peng M, Jin Z, et al. BACH1 is critical for homologous recombination and appears to be the Fanconi anemia gene product FANCJ. Cancer Cell 2005;8:255-265.

24. Meetei AR, DeWinter JP, Medhurst AL, et al. A novel ubiquitin ligase is deficient in Fanconi anemia. Nat Genet 2003;35:165-170.

25. Meetei AR, Medhurst AL, Ling C, et al. A human ortholog of archaeal DNA repair protein Hef is defective in Fanconi anemia complementation group M. Nat Genet 2005;37:958-963.

26. Reid S, Schindler D, Hanenberg H, et al. Biallelic mutations in PALB2 cause Fanconi anemia subtype FA-N and predispose to childhood cancer. Nat Genet 2007;39:162-164.

27. Xia B, Dorsman JC, Ameziane N, et al. Fanconi anemia is associated with a defect in the BRCA2 partner PALB2. Nat Genet 2007;39:159-161.

28. Pulsipher M, Kupfer GM, Naf D, et al. Subtyping analysis of Fanconi anemia by immunoblotting and retroviral gene transfer. Mol Med 1998;4:468-479.

29. Hanenberg H, Batish SD, Pollok KE, et al. Phenotypic correction of primary Fanconi anemia $\mathrm{T}$ cells with retroviral vectors as a diagnostic tool. Exp Hematol 2002;5:410-420.

30. Levran O, Diotti R, Pujara K, Batish SD, Hanenberg H, Auerbach AD. Spectrum of sequence variations in the FANCA gene: an International Fanconi Anemia Registry (IFAR) study. Hum Mutat 2005;25:142-149.

31. Chandra S, Levran O, Jurickova I, et al. A rapid method for retrovirusmediated identification of complementation groups in Fanconi anemia patients. Mol Ther 2005;12:976-984.

32. Casado JA, Callen E, Jacome A, Rio P. A comprehensive strategy for the subtyping of patients with Fanconi anaemia: conclusions from the Spanish Fanconi Anemia Research Network. J Med Genet 2007;44:241-249.

33. Buchwald M. Complementation groups: one or more per gene? Nat Genet 1995; 11:228-230.

34. Ianzano L, D'Apolito M, Centra M, et al. The genomic organization of the Fanconi anemia group A (FAA) gene. Genomics 1997;41:309-314.

35. Wijker M, Morgan NV, Herterich S, et al. Heterogeneous spectrum of mutations in the Fanconi anaemia group A gene. Eur J Hum Genet 1999;7: $52-59$

36. Morgan NV, Essop F, Demuth I, et al. A common Fanconi anemia mutation in black populations of sub-Saharan Africa. Blood 2005;105:3542-3544.

37. Magdalena N, Pilonetto DV, Bitencourt MA, et al. Frequency of Fanconi anemia in Brazil and efficacy of screening for the FANCA 3788-3790del mutation. Braz J Med Biol Res 2005;38:669-673.

38. Callén E, Tischkowitz MD, Creus A, et al. Quantitative PCR analysis reveals a high incidence of large intragenic deletions in the FANCA gene in Spanish Fanconi anemia patients. Cytogenet Genome Res 2004;104:341-345.

39. Yagasaki H, Hamanoue S, Oda T, Nakahata T, Asano S, Yamashita T. Identification and characterization of novel mutations of the major Fanconi anemia gene FANCA in the Japanese population. Hum Mut 2004;24:481490

40. Kutler DI, Auerbach AD. Fanconi anemia in Ashkenazi Jews. Fam Cancer 2004;3:241-248.

41. Bouchlaka C, Abdelhak S, Amouri A, et al. Tunisian Fanconi Anemia Study Group. Fanconi anemia in Tunisia: high prevalence of group A and identification of new FANCA mutations. J Hum Genet 2003;48:352-361.

42. Tamary H, Dgany O, Toledano H, et al. Molecular characterization of three novel Fanconi anemia mutations in Israeli Arabs. Eur J Haematol 2004;72: $330-335$

43. Savino M, Ianzano L, Strippoli P, et al. Mutations of the Fanconi anemia group A gene (FAA) in Italian patients. Am J Hum Genet 1997;61:12461253

44. Tachibana A, Kato T, Ejima Y, et al. The FANCA gene in Japanese Fanconi anemia: reports of eight novel mutations and analysis of sequence variability. Hum Mut 1999;13:237-244

45. Tamary H, Bar-Yam R, Shalmon L, et al. Fanconi anaemia group A (FANCA) mutations in Israeli non-Ashkenazi Jewish patients. Br J Haematol 2000; $111: 338-343$

46. Savino M, Borriello A, D'Apolito M, et al Spectrum of FANCA mutations in Italian Fanconi anemia patients: identification of six novel alleles and phenotypic characterization of the S858R variant. Hum Mut 2003;22:338-339.

47. Ameziane N, Errami A, Léveillé F, et al Genetic subtyping of Fancon anemia by comprehensive mutation screening. Hum Mut 2008;29:159-166.

48. Levran O, Erlich T, Magdalena N, et al. Sequence variation in the Fanconi anemia gene FAA. Proc Natl Acad Sci USA 1997;94:13051-13056.

49. Centra M, Memeo E, d'Apolito M, et al. Fine exon-intron structure of the Fanconi anemia group A $(F A A)$ gene and characterization of two genomic deletions. Genomics 1998;51:463-467.

50. The Fanconi anemia/breast cancer consortium. Positional cloning of the Fanconi anaemia group A gene. Nat Genet 1996;14:324-328.

51. Butturini A, Gale RP, Verlander PC, Adler-Brecher B. Hematologic abnormalities in Fanconi anemia: an International Fanconi Anemia Registry Study. Blood 1994;84:1650-1655.

52. Adachi D, Oda T, Yagasaki H, et al. Heterogeneous activation of the Fancon anemia pathway by patient-derived FANCA mutants. Hum Mol Genet 2002; $11: 3125-3134$.

53. Tachibana A. Mutational analyses of Fanconi anemia genes in Japanese patients. In: Shamim Ahmad, Sandra Kirk, editors. Molecular mechanisms of Fanconi anemia. New York: Springer, 2006:103-114.

54. Levran O, Doggett NA, Auerbach AD. Identification of Alu-mediated deletions in the Fanconi anemia gene FAA. Hum Mut 1998;12:145-152.

55. Morgan NV, Tipping AJ, Joenje H, Mathew CG. High frequency of large intragenic deletions in the Fanconi anemia group A gene. Am J Hum Genet 1999;65:1330-1341

56. Ausubel FJ, Brent R, Kingston RE, et al. Short protocols in molecular biology. New York: Wiley, 1992.

57. Cervenka J, Arthur D, Yasis C. Mitomycin C test for diagnostic differentiation of idiopathic aplastic anemia and Fanconi anemia. Pediatrics 1981;67:119-27.

58. Auerbach AD, Adler B, Chaganti RS. Prenatal and postnatal diagnosis and carrier detection of Fanconi anemia by a cytogenetic method. Pediatrics 1981;67:128-135.

59. Fanconi Anemia Mutation Database. Available at: http://www.rockefeller.edu/ fanconi/mutate/jumpa.html. Accessed September 2008

60. SNP linked to Gene FANCA (geneID:2175) Via Contig Annotation. Available at: http://www.ncbi.nlm.nih.gov/SNP/snp ref.cgi?locusId $=2175 \&$ chooseRs $=$ all\#17232623. Accessed February 2008 .

61. The Human Gene Mutation Database at the Institute of Medical Genetics in Cardiff. Available at: http://www.hgmd.cf.ac.uk/ac/all.php. Accessed February 2008

62. Fanconi Anemia database. Fanconi anemia, complementation group A Leiden Open Variation Database (LOVD). Available at: http://chromium.liacs.nl/ LOVD2/FANC/home.php?select_db=FANCA. Accessed September 2008

63. Pertea M, Lin X, Salzberg SL. GeneSplicer: a new computational method for splice site prediction. Nucleic Acids Res 2001;29:1185-1190. Available at: http://www.tigr.org/tdb/GeneSplicer/gene_spl.html.

64. Reese MG, Eeckman FH, Kulp D, Haussler D. Improved splice site detection in Genie. J Comp Biol 1997;4:311-323. Available at: http://www.fruitfly.org/ seq_tools/splice.html.

65. Brunak S, Engelbrecht J, Knudsen S. Prediction of human mRNA donor and acceptor sites from the DNA sequence. J Mol Biol 1991;220:49-65. Available at: http://www.cbs.dtu.dk/services/NetGene2/.

66. Rogan PK, Schneider TD. Using information content and base frequencies to distinguish mutations from genetic polymorphisms in splice junction recognition sites. Hum Mut 1995;6:74-76.

67. Ng PC, Henikoff S. Accounting for human polymorphisms predicted to affect protein function. Genome Res 2002;12:436-446. Available at: http://blocks. fhcrc.org/sift/SIFT.html.

68. Ramensky V, Bork P, Sunyaev S. Human non-synonymous SNPs: server and survey. Nucleic Acids Res 2002;30:3894-3900. Available at: http:/genetics. bwh.harvard.edu/pph/.

69. Poole SR, Smith AC, Hays T, McGavran L, Auerbach AD. Monozygotic twin girls with congenital malformations resembling fanconi anemia. Am J Med Genet 1992;42:780-784

70. Mankad A, Taniguchi T, Cox B, et al. Natural gene therapy in monozygotic twins with Fanconi anemia. Blood 2006;107:3084-3090.

71. Gualandi F, Neri M, Bovolenta M, et al. Transcriptional behavior of DMD gene duplications in DMD/BMD males. Hum Mutat 2008;29:E310-E319. 